\title{
Les dictionnaires monolingues généraux du fran- çais "actuel” gratuits en ligne (début 2019)
}

\author{
Pierre Corbin ${ }^{1 *}$, et Nathalie Gasiglia ${ }^{2}$ \\ ${ }^{1}$ UMR 8163 (Savoirs, Textes, Langage) - Université de Lille, France \\ ${ }^{2}$ UMR 8163 (Savoirs, Textes, Langage) - Université de Lille, France
}

\begin{abstract}
Résumé. Cet article présente une vue d'ensemble des dictionnaires généraux en ligne gratuits du français actuel au début de 2019, en mettant l'accent sur leur nature et leur origine.
\end{abstract}

\begin{abstract}
Free online general monolingual dictionaries of current French: an overview (early 2019) This paper presents an overview of free online general dictionaries of current French at the beginning of 2019, focusing on their nature and their origins.
\end{abstract}

\section{Dictionnaires payants, dictionnaires gratuits}

Demande et offre de dictionnaires monolingues généraux fluctuent en fonction d'évolutions sociales et technologiques. Naguère, acheter un grand dictionnaire en plusieurs volumes, surtout s'il était "encyclopédique", pouvait être considéré comme un placement intellectuel susceptible de soutenir le devenir scolaire des enfants. Internet advint et ce monde disparut (cf. Corbin 2008). Nous voulons désormais des réponses gratuites immédiates à des questions simples - comment ça s'écrit ? qu'est-ce que ça veut dire ? comment ça se conjugue ? -, et normalement nous les obtenons en ligne, sans souci de qui nous les fournit et où nous les trouvons. Et il nous est aussi possible d'intervenir dans l'élaboration de ces réponses en nous improvisant lexicographes sur des sites dits collaboratifs, contributifs ou participatifs selon les modalités de l'encadrement des apports individuels.

Dans cet environnement nouveau perdure cependant - en France tout au moins, et pour combien de temps encore ? - un commerce de dictionnaires imprimés et/ou électroniques élaborés par des lexicographes professionnels.

Quatre maisons d'édition - Larousse, Le Robert, Hachette Éducation, subsidiairement Auzou - se partagent inégalement l'essentiel de l'offre imprimée, quelques éditeurs de livres scolaires ou de jeunesse s'ajoutant à eux pour proposer divers répertoires pour enfants. Stratification et récurrence sont les caractéristiques de cette offre : les catalogues des éditeurs majeurs se structurent, hormis quelques produits de niche, en trois ensembles - les dictionnaires de référence, "de langue" ou "encyclopédiques", désormais plafonnés à un volume unique ; les utilitaires (usuellement dénommés "pratiques"), déclinés dans les formats "maxi", "poche", "mini", voire "micro", et proposant des données de richesse échelonnée ; et les scolaires (pour le collège, les cycles de l'école élémentaire et, à un degré moindre, la maternelle) -,

*pierre.corbin@univ-lille.fr. 
tous ces ouvrages étant régulièrement réédités - certains avec un millésime annuel -, sans profonds changements mais avec une apparence plus ou moins renouvelée et, pour les plus notoires, une campagne de promotion des innovations infimes (cf. Corbin 1991, 1998, 2002, 2008, et Corbin \& Corbin 2008).

Il en va autrement de l'offre numérique payante, dans laquelle, hors versions bimédia de certains dictionnaires imprimés, seul Le Robert est aujourd'hui pleinement investi, avec un choix diversifié d'abonnements en ligne, de téléchargements pour ordinateurs, d'applications pour tablettes ou smartphones et d'e-books pour liseuses, Larousse se limitant à un nombre réduit d'applications, tandis qu'Hachette Éducation et Auzou ne proposent aucun produit de cette nature.

Face à cette production marchande, l'offre numérique gratuite en ligne apparaît comme abondante, hétérogène, non structurée, inégalement et plutôt médiocrement documentée, difficile à cerner dans sa globalité et évolutive. L'objet de cette contribution est d'en présenter un état à un moment déterminé (le début de l'année 2019), afin de garder une trace datée de l'offre qui puisse servir de repère comparatif pour l'observation des évolutions ultérieures, déjà perceptibles au moment de cette rédaction (début 2020).

\section{Méthode de recensement des dictionnaires généraux du fran- çais "actuel" gratuits en ligne}

Pour dresser un inventaire le moins lacunaire possible des répertoires consultables gratuitement en ligne susceptibles d'être considérés comme des dictionnaires généraux du français "actuel", il faut associer tous les moyens empiriques disponibles.

Commencer en usager aléatoire cherchant un dictionnaire ou une information sur un item avec des moteurs de recherche apporte déjà une moisson abondante, certes dépendante du référencement des sites, mais sujette à s'amplifier si on fait varier la forme des requêtes (dictionnaire + français / usuel / monolingue / unilingue / général / de langue ... ; item $x$ / y / z ... + définition / sens...) et les navigateurs utilisés, et qu'on contrôle l'historique des recherches. À titre illustratif, deux requêtes effectuées le 20 octobre 2018 avec Safari depuis un iPhone 7 sur les motifs dictionnaire français et reculer définition ont fourni, cumulativement, une vingtaine de résultats pertinents - respectivement une douzaine et une quinzaine regroupés dans les trente-cinq et cinquante premiers de listes qui cessaient ensuite d'être productives, avec des intersections importantes entre elles mais aussi des spécificités dans chacune.

Chemin faisant, on découvre des annuaires de dictionnaires (cf. Annexe 1), de qualité et de rendements très variables, le plus performant, Lexilogos (sur lexilogos.com), figurant ordinairement parmi les premiers résultats d'une requête de la forme dictionnaire français / de langue. Ce portail, qui recense des ressources pour de nombreuses langues, redirige, pour le français "actuel”, vers une quinzaine de dictionnaires généraux a priori pertinents répartis sur un nombre comparable de sites, en ciblant au choix, quand c'est possible, une interface de consultation et/ou leur page d'accueil, ou, par la saisie d'un motif, un article particulier. Le recouvrement avec les résultats des requêtes évoquées est important mais pas intégral, Lexilogos donnant spécifiquement accès au Grand Larousse de la langue française [GLLF] numérisé sur gallica.bnf.fr (cf. § 5.1.2) et à la compilation du site russe Academic titrée Encyclopédie universelle sur fracademic.com, non repérée par des investigations avec des moteurs de recherche et qui paraît contrevenir au respect de la propriété intellectuelle (cf. $\S 5.1 .1$ et 5.1.2). Certaines pages de Wikipédia (cf. Annexe 2) contribuent aussi au repérage, en jouant les mêmes rôles d'annuaire et de redirection vers des sites de consultation ou en menant à d'autres pages documentaires de la même encyclopédie. Et l'inventaire peut encore être enrichi par la connaissance préalable de sites non détectés par les voies précédentes ou par le hasard de recherches sur Internet, ce qui est un indice de l'incomplétude probable de la glane effectuée. 


\section{Identification et sélection des dictionnaires}

À la compilation d'URL succèdent des investigations visant à déterminer à quelle(s) version(s) de quels dictionnaires elles permettent d'accéder et quel est le degré d' "actualité" de cellesci. La tâche n'est pas aisée, et beaucoup de réponses font encore défaut, pour plusieurs raisons.

D'abord, les sources de l'offre sont de diverses natures : des dictionnaires imprimés institutionnels ou commerciaux, structurés informatiquement ou seulement numérisés, en constituent une part ( $\$ 5.1)$; certains autres sont proposés par des entreprises développant des outils informatiques pour le traitement automatique des langues, l'aide à l'expression ou la correction orthographique (\$ 5.2); d'autres encore ont une origine difficilement identifiable (§ 5.3) ; plusieurs sont, à des degrés différents, collaboratifs ou participatifs (§ 5.4); et un au moins est une création universitaire originale pour le français langue étrangère (§ 5.5).

Ensuite, les sites de consultation des dictionnaires repérés sont soit ceux de leurs producteurs, soit ceux d'hébergeurs (contractuels ou non), d'où il découle qu'un même répertoire ou des versions différentes de celui-ci soient accessibles sur plusieurs sites.

D'autre part, le traitement modulaire des types de contenus d'articles de dictionnaires imprimés, courant sur Internet, peut être une source d'incertitude quant à ce qu'il faut ou ne faut pas prendre en compte : alors que, s'agissant d'ouvrages imprimés, on intégrerait un dictionnaire d'expressions dans les répertoires spécialisés en l'excluant de l'ensemble des dictionnaires généraux, l'autonomisation, en ligne, de la consultation des expressions, par exemple via un bouton ou un onglet spécifique, peut résulter de l'affichage séparé de différents composants des articles d'un dictionnaire général, sans pourtant que ce soit toujours le cas.

En effet, à l'inverse, une même interface de consultation peut associer des ressources originellement indépendantes et possiblement dépareillées (par exemple un dictionnaire "de langue" et un "dictionnaire" de citations (qui n'est en général qu'une anthologie non métalinguistique pouvant être organisée de différentes façons), ce qui, pour la sélection des répertoires pertinents, est une difficulté symétrique de la précédente.

Un autre obstacle, majeur, tient à l'insuffisance des métadonnées pour divers dictionnaires, concernant tant leurs origines, les versions proposées et leur datation que leurs contenus, et donc leur "actualité".

Par ailleurs l'évaluation de celle-ci, qui a une dimension théorique et une dimension pratique, est en elle-même un critère décisif d'appréciation de l'appartenance des dictionnaires repérés à l'ensemble à définir :

- Dans son principe, cette problématique, qui n'est pas propre aux dictionnaires en ligne (gratuits ou payants), comporte deux volets : jusqu'à quel recul dans le passé un dictionnaire peutil être considéré comme traitant encore du français "actuel", et quel degré d'actualisation doit-il présenter pour ne pas être frappé d'obsolescence ? Pour chaque question une certaine souplesse d'appréciation est nécessaire :

- concernant son recul diachronique, on peut estimer qu'un dictionnaire reste "actuel" s'il intègre le vocabulaire passif des locuteurs âgés, ce qui autorise une couverture de l'ordre du siècle (cf. Rey-Debove 1971 : 95);

- s'agissant de sa modernité, on peut considérer qu'un dictionnaire qui n'enregistrerait pas les mots ou expressions entrés le plus récemment en usage ne serait pas pour autant "inactuel", mais qu'il en irait autrement si des ensembles lexicaux cohérents et importants, relevant par exemple de terminologies de domaines en pointe, faisaient défaut (ce qui est d'ailleurs le cas pour certains dictionnaires généraux imprimés encore commercialisés, comme le Lexis de Larousse, dont la nomenclature, fixée en 1975, n'a pas été significativement revue).

- En pratique, l'appréciation de l'“actualité" des dictionnaires inventoriés ne peut se faire qu'au cas par cas, en fonction des connaissances accessibles, qui sont très variables : 
- des dictionnaires déjà un peu anciens et non susceptibles d'être mis à jour vont s'éloigner toujours plus du lexique "actuel" : c'est le cas au premier chef du Trésor de la langue française informatisé [TLFi] et du GLLF (cf. §§ 5.1.1 et 5.1.2) ;

- d'autres sont explicitement ancrés dans le présent, à l'instar de la 9 e édition du Dictionnaire de l'Académie française [DAF9], encore sur le métier et pour laquelle un addenda à ce qui a été rédigé depuis le premier fascicule de 1986 est consultable sur le site de l'institution (cf. $§ 5.1 .1$ );

- pour certains dictionnaires accessibles sur plus d'un site, des variations dans les indications quantifiées fournies, dans les dates de copyright ou dans les données observées suggèrent qu'il s'agisse de versions inégalement riches échelonnées dans le temps : il en va ainsi pour le Dictionnaire Cordial [DCor] et pour le dictionnaire sensAgent [sA] (cf. $\S \S 5.2 .1$ et 5.2.2) ;

- mais, pour la plupart des répertoires, leur degré d' "actualité" lexicale reste à évaluer empiriquement par la confrontation de leur nomenclature avec divers inventaires de "mots nouveaux", d'entrées récentes dans des dictionnaires commerciaux et d'attestations documentées dont on dispose par ailleurs.

\section{Diversité de l'inventaire provisoire}

Vingt-trois dictionnaires ont été recensés, distribués sur vingt-huit sites. Cet ensemble est hétérogène à divers égards :

- l'identification éditoriale des répertoires : de qui (institutions, entreprises, auteurs) émanentils ? ont-ils des sources et lesquelles ? de quand datent-ils ? Les réponses à ces questions peuvent être fournies sur les sites de consultation, ou connaissables de manière indépendante, ou encore indéterminables ;

- les informations quantitatives et qualitatives sur les contenus : comme les informations précédentes, celles qui concernent l'ordre de grandeur des nomenclatures et la nature des données fournies peuvent se trouver dans des métadonnées explicites, ou requérir des investigations (ce qui est toujours faisable pour l'identification qualitative des contenus), ou être inaccessibles (ce qui est le cas pour une part significative des données quantitatives);

- la sélection des composants des articles : d'ampleur variable, le choix de ces rubriques puise le plus souvent dans celles qui sont classiquement mises en œuvre dans les dictionnaires monolingues généraux, mais il peut également intégrer des données inédites, frôlant parfois le gadget, à l'instar des nuages de mots du dictionnaire français d'educalingo.com [df-educa] (cf. § 5.4), ou des traductions, comme celles, en anglais, du Dictionnaire français de linternaute.com [DF-LInt] (cf. § 5.3);

- l'affichage des informations, qui donne lieu à plusieurs modes de présentation :

- la reproduction des articles de dictionnaires imprimés proposés en consultation électronique, qui se rencontre, par définition, quand l'interface est un fac-similé de la source résultant d'une numérisation, ce qui est le cas pour le $G L L F$ (cf. $\S \S 2$ et 5.1.2) ;

- le calque de la forme des articles des sources imprimées, qui garde leur linéarité en modifiant leur présentation par l'application d'une feuille de styles plus ou moins spécifique, comme dans le TLFi (cf. § 5.1.1), et, dans le meilleur des cas, un "design adaptatif" préservant le confort de consultation sur tous types d'écrans (et notamment ceux des smartphones, considérés désormais comme les supports les plus utilisés et les plus universellement accessibles), par exemple dans l'interface récente du DAF9 (ibid.) sur dictionnaireacademie.fr (cf. son « Dossier de presse » de février 2019, pp. 15 et 18);

- 1'association de la linéarité et de la modularité dans des dictionnaires comme le DCor sur cordial.fr/dictionnaire/ ou le $d f$-educa (cf. $\S \S 5.2 .1$ et 5.4), qui, tout en gardant une 
présentation linéaire du texte des articles, le séquencent en modules bien séparés (parfois par des publicités dans le second) et étiquetés, à la façon d'un état de requête de base de données ;

- ou, dans d'autres dictionnaires, une modularisation plus radicale des données, rompant avec leur enchaînement linéaire pour mener à celles-ci par des liens et des boutons sélectionnant des emplois et des rubriques, comme dans le Dictionnaire de français [DdFLar] sur larousse.fr (cf. § 5.1.2), voire par des changements de sites, à l'instar, pour le dictionnaire.net $[$ d.n] , de dictionnaire.net, synonymes.net et conjugaison.net (cf. § 5.2.3) ;

- l'offre, clairsemée et pluriforme, de fonctionnalités propres à l'environnement électronique :

- recherches paramétrables dans le texte intégral du TLFi et du DAF9, respectivement sur atilf.atilf.fr et academie.atilf.fr seulement ;

- surlignage paramétrable de composants balisés des articles du TLFi sur atilf.atilf.fr et cnrtl.fr/definition/ ;

- regroupements d'articles préparamétrés dans le $D F$-LInt pour les items de même catégorie, de même registre ou de même domaine (cf. § 5.3) ;

- liens, dans les articles appropriés du DAF9 (cf. § 5.1.1) sur dictionnaire-academie.fr seulement, vers des ressources lexicales parentes internes (Dire, ne pas dire) ou externes (FranceTerme, Base de données lexicographiques panfrancophone).

\section{Classement provisoire}

Le classement qui suit répartit les dictionnaires recensés au moment de l'étude, en fonction de leur source, dans les cinq ensembles distingués au début du $§ 3$. D'autres classements concevables ne sont pas réalisables actuellement, par exemple une mise en parallèle avec les catégories utilisées dans les catalogues des éditeurs commerciaux de dictionnaires imprimés (cf. $\S 1$ ), qui suppose de mieux connaître les caractéristiques quantitatives et qualitatives de chaque répertoire (cf. $\S \S 3$ et 4 ). L'emploi de l'imparfait signale une évolution entre le moment de l'étude et celui de la rédaction (cf. $\S 1$ ), tandis que le présent connote la stabilité. L'Annexe 3 liste les URL pertinentes pour chaque dictionnaire sur chaque site.

\subsection{Dictionnaires émanant de dictionnaires imprimés}

Dix dictionnaires - deux institutionnels et huit commerciaux - procèdent de répertoires imprimés. Leurs accès sont répartis sur neuf sites, sans relations biunivoques entre dictionnaires et sites.

\subsubsection{Dictionnaires institutionnels}

Les deux dictionnaires institutionnels, bien documentés sur certains sites officiels, sont le $T L F i$ et le DAF9.

- Le Trésor de la langue française informatisé

Ce dictionnaire "de langue" de référence en ligne depuis 2002 produit par le laboratoire ATILF sur la base des seize volumes du TLF imprimé de 1971-1994, qui a vocation à couvrir et décrire dans ses diverses dimensions le lexique du français entre 1789 et 1960 et n'est donc pas évolutif, est repéré sur quatre sites :

- Deux seulement sont officiels (atilf.atilf.fr et cnrtl.fr/definition/), avec des fonctionnalités différentes déjà évoquées, atilf.atilf.fr étant celui qui permet d'effectuer des investigations poussées dans le texte intégral (cf. § 4). Ce site informe aussi sur l'ordre de grandeur de plusieurs ensembles d'éléments constitutifs du dictionnaire : « 100000 mots avec leur histoire », « 270000 définitions », « 430000 exemples » et « 350 millions de caractères ». 
- Curieusement, le site privé le-tresor-de-la-langue.fr, dont une large part des métadonnées a été supprimée, qui assumait d'être «illégal en terme[s] de propriété intellectuelle» (« À Propos ») et dont la vocation explicitée par son développeur était, sans but lucratif, de donner à lire le $T L F i$ « sous un meilleur jour » (désormais dans « une meilleure version »), avec une présentation modernisée et une recherche directe en plein texte, n'évoquait, selon les pages, que «49000 entrées principales » ou «50 000 entrées » et « environ 95000 définitions » (actuellement « environ 50000 définitions de mots »), écart important avec les données officielles qui ne se laisse pas interpréter a priori.

- Les articles du TLFi sont aussi reproduits sur fracademic.com sans mention de leur origine (cf. § 2), leurs contenus y étant regroupés avec ceux du DdF-Lar, de deux dictionnaires Robert et d'un dictionnaire Hachette sous la dénomination globale d'Encyclopédie universelle, assortie de la date 2012 (cf. § 5.1.2).

- La $9^{e}$ édition du Dictionnaire de l'Académie française

Dictionnaire d'usage pour l' "honnête homme", donc d'une "actualité" raisonnée, le DAF9, en voie d'achèvement (les trois volumes parus en 1992, 2000 et 2011 couvrent le lexique de $a$ à quotité, et la rédaction a depuis atteint savoir), est lui aussi hébergé sur atilf.fr (depuis 2000), avec un affichage récemment rénové (academie.atilf.fr, accessible également depuis cnrtl.fr/dictionnaires/modernes/), et, désormais, dans une version optimisée pour smartphones, sur dictionnaire-academie.fr (cf. § 4). Très bien documenté, sur ce site, par sa « Présentation détaillée », son « Aide en ligne », ses « Préfaces et annexes du Dictionnaire » et son «Dossier de presse » de février 2019, qui exposent sa vocation, les principes de son élaboration et les modalités de sa consultation, le $D A F 9$ devrait couvrir à terme une nomenclature évaluée, selon les paratextes, à « 55000 mots » ou « entre 55000 et 60000 mots », traduisant, par rapport à celle de la $8^{\mathrm{e}}$ édition [DAF8], un accroissement «d'environ 25000 mots » ou de «plus de 25000 mots », et équivalant à l'ordre de grandeur de celle du $P R$ ou de la composante "de langue" du Petit Larousse illustré $[P L I]$. La possibilité déjà offerte de consulter les $8^{\mathrm{e}}$ et $9^{\mathrm{e}}$ éditions du $D A F$ à partir d'une «nomenclature fusionnée » de «près de 56000 mots » («Dossier de presse », p. 16) s'est étendue depuis décembre 2019 aux sept éditions antérieures, pour une « super-nomenclature » globale de «plus de 250000 articles » (Id., p. 14).

\subsubsection{Dictionnaires commerciaux}

Trois des huit dictionnaires commerciaux émanent de Larousse, trois du Robert, un de Hachette et un du producteur de contenus lexicaux israélien K Dictionaries.

- Les dictionnaires Larousse

Les trois dictionnaires Larousse consultables en ligne, dont un seul sur le site de l'éditeur, diffèrent fortement tant par leur facture que par les conditions de leur consultation :

- Le GLLF, dictionnaire "de langue" de référence publié en sept volumes de 1971 à 1978, non pérennisé et donc vieillissant (cf. § 3), est accessible sur gallica.bnf.fr sous la forme du fac-similé d'une réédition de 1989 (cf. $\$ \$ 2$ et 4), la consultation s'effectuant volume par volume, avec une fiche de métadonnées détaillée pour chacun d'eux et une possibilité de recherche de motifs en texte intégral lourde et imprécise.

- Le $D d F$-Lar, dont le site larousse.fr, qui le propose, n'explique pas la genèse, est une version évoluée de la composante "de langue" du Grand Larousse en 5 volumes, dictionnaire "encyclopédique" publié en 1987, via le Grand usuel Larousse en 5 volumes de 1997 et des exploitations numériques sur disques. Sa nomenclature, accessible par un index à partir d'un lien en bas des pages, est d'environ 83000 entrées, et la page d'accueil faisait état, au moment de l'étude, de « 135000 définitions », « 34000 expressions », « 92000 synonymes », « 29000 contraires », « 15000 homonymes » et « 6000 articles » traitant de difficultés linguistiques. En revanche, les modalités et la portée de son actualisation restent à déterminer (covoiturage, écoparticipation, écotaxe, flashcode, hameçon- 
nage, relou, selfie, smartphone, sudoku ou vapoter y étaient traités, mais pas phablette, QR Code ou vénère, enregistrés dans le $P L I$ et dont les deux premiers n'ont été intégrés que plus récemment dans le $D d F$-Lar). La présentation des données, désormais en partie linéaire, était modulaire (cf. § 4), aussi bien pour les adresses, à raison d'une pour chaque association d'un item et d'une catégorisation, que pour les informations, issues de plusieurs sources et pas toutes métalinguistiques, réparties entre les rubriques « Définitions », «Expressions », « Synonymes », « Homonymes », « Difficultés » et « Citations », auxquelles on accédait par des onglets. Il est à noter qu'il existe également des applications payantes du DdF-Lar pour iOS et Android. Des contenus de ses articles antérieurs à 2012 sont en outre reproduits purement linéairement sur fracademic.com dans les mêmes conditions que ceux du TLFi (cf. $\S \S 2$ et 5.1.1).

- Enfin, le Maxipoche 2014 [Mp], consultable, parmi d'autres dictionnaires, sur le portail fr.thefreedictionary.com développé par la société américaine Farlex, reproduit, sans fonctionnalités ajoutées et avec une nouvelle mise en forme, les articles de l'édition de 2013 d'un dictionnaire utilitaire de milieu de gamme remillésimé annuellement, pour lequel la version imprimée source fait état de « plus de 40000 mots », « 10000 noms propres », environ « 75000 définitions » et « 50000 exemples ».

- Les dictionnaires Robert

- À la différence de Larousse, Le Robert ne proposait pas d'accès gratuit à un dictionnaire électronique avant début avril 2020. On est donc fondé à penser que la reproduction d'articles du Grand Robert de la langue française [GRLF] et du Petit Robert $[P R]$ sans datation (il pourrait s'agir, pour ce dernier, d'une version de 1996) sur fracademic.com, dans les conditions déjà évoquées pour le TLFi (\$ 5.1.1) et le DdF-Lar (supra) et avec perte des fonctions de recherche, ne résulte pas d'un accord avec l'éditeur.

- Il en va autrement pour les articles que Google affiche couramment comme première réponse à des requêtes sur des motifs lexicaux, qui adaptent, sans explicitation mais en partenariat avec l'éditeur, une version de la composante "de langue" du Robert illustré $[R I$ intégrée au Robert correcteur (cf. Gasiglia à paraître a et b). Si l'actualisation du dictionnaire source est commensurable à celle du $D d F$-Lar, les réponses spontanées de Google ne la répercutent pas intégralement (distorsions, par exemple, pour flashcode et phablette).

- Le dictionnaire Hachette

C'est encore dans l'environnement évoqué de fracademic.com qu'on peut consulter, sans davantage d'identification et avec perte de sa mise en forme, le Dictionnaire Hachette. Langue française et synonymes électronique de 2002, dont une source était le Dictionnaire Hachette langue française imprimé (dernière édition en 2001, 40000 mots selon sa couverture).

- Le dictionnaire de K Dictionaries

Le dictionnaire sans titre au copyright de 2013 produit par K Dictionaries $[d-K]$ dont on trouve les articles sur fr.thefreedictionary.com dans les mêmes conditions que le $M p$ Larousse est un petit répertoire généré de façon automatisée qui associe des traductions en anglais et des définitions brèves précédant des exemples provenant de la matrice française de dictionnaires bilingues imprimés de poche diffusés en France par Assimil depuis 2009.

\subsection{Dictionnaires émanant de sociétés de technologies linguistiques}

Trois dictionnaires, répartis sur neuf sites, sont proposés par des sociétés d'ingénierie linguistique. 


\subsubsection{Le Dictionnaire Cordial de Synapse Développement}

On peut consulter sur au moins quatre sites des versions du DCor produit par l'entreprise toulousaine Synapse Développement, créatrice du logiciel de correction Cordial :

- Sur cordial.fr/dictionnaire/ sont annoncées « 216000 entrées lexicales pour 310000 définitions » couvrant « le vocabulaire général, ainsi que les termes les plus communs des principaux domaines scientifiques et techniques ». Les données associées aux adresses sont traitées dans une succession linéaire de modules étiquetés (cf. § 4) : définitions, expressions, difficultés, synonymes, contraires, attestations d'emplois, mots proches. L'actualisation périodique de la nomenclature (en octobre 2018, le site ne mentionnait que «153 000 entrées lexicales pour 190000 définitions ») paraît consistante, à en juger par l'intégration de tous les mots récents évoqués au titre de leur attestation dans le $D d F$-Lar et de certains qui en étaient encore absents (phablette, vénère).

- Sur le site journalistique la-conjugaison.nouvelobs.com, le paratexte d'accueil auquel mène le lien «Définition» du menu évoque une nomenclature de «plus de 150000 mots ». Les données, moins diverses et entourées de publicités, se distribuent sous les boutons « Définition » (qui donne accès aux « Expressions » après les définitions) et « Synonyme » (sous lequel synonymes et antonymes sont regroupés dans des subdivisions numérotées, alors que le DCor de cordial.fr/dictionnaire/ les disjoint et n'utilise pas de numérotation). L'actualisation de la nomenclature, limitée à covoiturage, écotaxe et sudoku pour l'échantillon testé, n'est pas commensurable à celle de la version de référence.

- Le menu du site encyclopédique universalis.fr propose, sous « Dictionnaire », une version du DCor encore plus réduite (" plus de 122000 entrées » d'après l'écran d'accueil) et limitée à des définitions de mots et d'expressions enchainées linéairement et dépourvues de codifications typographiques, dans une interface exempte des publicités présentes sur le site global de l'encyclopédie et qui donne accès à la nomenclature ordonnée alphabétiquement par le lien « Consulter le dictionnaire de l'Encyclopædia Universalis » en bas de l'écran. Covoiturage et écotaxe sont les seuls mots récents testés qui y figurent, ce qui est encore moins que sur la-conjugaison.nouvelobs.com.

- Le site dictionnairique dictionnaire.reverso.net, qui panache diverses ressources, crédite le DCor pour des définitions de mots (mais pas d'expressions) et des synonymes et contraires (réunis en ensembles numérotés comme sur la-conjugaison.nouvelobs.com). En l'absence de métadonnées, on peut estimer que sa couverture lexicale soit intermédiaire entre celles de cordial.fr/dictionnaire/ et de la-conjugaison.nouvelobs.com, puisqu'elle inclut covoiturage, écoparticipation, écotaxe, hameçonnage, smartphone et sudoku. Mais, via une autre ressource, son Dictionnaire collaboratif, le site apporte aussi des informations sur différents mots récents absents de la version du DCor utilisée (box (féminin), chelou, flashcode, hashtag, phablette, relou, selfie, seum, vapoter, vapotage, vapoteur, euse, vénère, wesh (interjection)).

\subsubsection{Le dictionnaire sensAgent de Sensegates}

Concernant le dictionnaire $s A$, consultable dans quatre interfaces sur trois sites, dont deux sous ce nom, on ne dispose, en guise de métadonnées, que de l'identification de la société qui le produit actuellement - Sensegates SARL, spécialisée dans le «traitement automatique des langues » et la « sémantique lexicale » -, à laquelle on accède via le lien « Informations » en bas d'écran des deux sites sensAgent. L'une des interfaces du troisième site, tv5monde. com, mentionne la plus ancienne société Memodata parmi les contributeurs, mais l'indication « Conçu et édité par sensagent » y redirige vers dictionnaire.sensagent.leparisien.fr. Les trois sites diffèrent par leurs interfaces et leur datation, mais puisent, avec des variations, dans le même ensemble de ressources : 
- Sur traduction.sensagent.com, le site sensAgent - traduction du développeur, daté par un « Copyright (C) 2013 sensagent », donne accès, en sélectionnant le français comme « langue source » et comme « langue cible » (« (C) $2015 »)$, à des données monolingues composites « définitions », « synonymes », « voir aussi » (= mots proches à divers titres), « locutions », « dictionnaire analogique » (organisé en thésaurus), « conjugaison» (= flexion non limitée aux verbes)..., mais aussi articles du Littré et de Wikipédia -, enchaînées pour certaines linéairement et toutes consultables via des boutons.

- Sur le site de presse dictionnaire.sensagent.leparisien.fr, assortie d'un « Copyright (C 20002016 sensagent », l'interface monolingue sensAgent - dictionnaire, visuellement proche de la précédente, s'affiche directement, avec un contenu similaire, quelques variations de boutons et la possible insertion de publicités. Des trois sites où $s A$ est consultable, celui-ci apparaît prioritairement dans les réponses aux requêtes de définitions avec des moteurs de recherche.

- Le site médiatique tv5monde.com donne accès à deux interfaces de $s A$ aux contenus non équivalents :

- La plus ancienne, enfouie dans l'architecture du site et avec pour seul repère chronologique un «Copyright 2003 » pour Wordnet 2.0, est celle d'un Traducteur multilingue - sous-titré Définitions et traductions avec Alexandria - où il faut sélectionner deux fois le français, comme sur traduction.sensagent.com, pour accéder aux résultats monolingues, modularisés similairement avec quelques nuances (notamment un bouton « dérivés » au lieu de « voir aussi »).

- L'autre, sur langue-francaise.tv5monde.com, invitant à Rechercher un mot dans le dictionnaire soit en cliquant sur une icone en haut à droite des pages d'accueil et «Découvrir le français » (sous «Langue française »), soit en bas de cette page », ne comporte ni identifiant ni datation et est restreinte à « définition », « synonyme » et « conjugaison ».

Faute d'informations sur le contenu dictionnairique spécifique de ces différentes versions, leur couverture lexicale ne peut être évaluée sans investigations. On observe cependant, par des requêtes sur les items aléatoires récemment apparus déjà exploités, que les trois premières versions évoquées sont actualisées similairement (covoiturage, écotaxe, selfie, smartphone, vapoter, wesh (interjection), yes) et que, pour d'autres mots, elles exploitent identiquement des définitions de Wikipédia (Code QR, flashcode, hameçonnage, hashtag, sudoku), alors que la nomenclature de la dernière version, qui ne traite aucun item testé, ne bénéficie pas des mêmes mises à jour.

\subsubsection{Les dictionnaires de Blue Painter}

La société iséroise de «programmation informatique » Blue Painter propose sur deux sites ce qui a l'apparence de variantes d'affichage d'un même répertoire, respectivement avec et sans publicité : Le Dictionnaire [ $L D]$ sur le-dictionnaire.com et $d . n$ sur dictionnaire.net, le premier apparaissant préférentiellement au second dans les résultats de requêtes lexicales sur Internet. Pour toutes métadonnées, les pages qui explicitent leurs conditions d'utilisation indiquent que « [1] es informations et données contenues dans [ces dictionnaires] sont élaborées via une base de données préalablement éditée » et qu' « [e]lles proviennent de dictionnaires ou d'ouvrages sur la langue française et illustrent les différentes possibilités de la langue en matière de synonymie ou d'antonymie par le biais de ces références », ce qui n'a pour but explicite que de dégager la responsabilité de la société vis-à-vis de ses contenus, sans aucunement renseigner sur ceux-ci. La couverture lexicale de ces dictionnaires n'est donc pas évaluable, et il en va de même pour l'actualisation du lexique recensé, pas inexistante mais assez restreinte au vu de sondages aléatoires (box (féminin), covoiturage, sudoku, mais pas écoparticipation, flashcode, hameçonnage, hashtag, selfie, smartphone, vapoter). Quant aux données fournies sur les mots répertoriés, elles se limitent à des définitions, à la conjugaison des verbes et à des synonymes, en inventaires séparés ouvrant autant de nouveaux sites, dont 
les contenus sont soit similaires, soit dans une relation inclusive (synonymes.com, dictionnairesynonymes.com, les-synonymes.com, synonymes.net).

\subsection{Dictionnaires d'origine indéterminée}

Trois dictionnaires, sur trois sites, dont deux dédiés à des types d'informations intéressant le grand public, peuvent être réunis par défaut, sur la base de leur origine documentaire non identifiée et de leur caractère non collaboratif.

\section{- Le dictionnaire Mediadico}

Le dictionnaire sans titre ni mentions légales accessible en sélectionnant « Dictionnaire » dans le menu «Loisirs » sur notrefamille.com (site de Bayard Presse destiné aux parents) enchaîne linéairement, sous le bouton « Définition », des définitions et synonymes qui lui sont propres, titrés « Définition et Synonymes - Définition Mediadico » (dernière trace de son nom d'origine), et des articles du Littré et du DAF8, et donne accès par d'autres boutons à la « Conjugaison » et à la «Traduction » (en anglais). Dépourvu de métadonnées, Mediadico [Md], encore doté en octobre 2018 d'un site propre devenu introuvable, est présenté par un article spécifique de Wikipédia comme remontant au 3615 Dico sur Minitel de 1987, dont les contenus étaient déjà assez sommaires (cf. Corbin 1991 : 12). La tentative d'évaluation de l'ordre de grandeur de sa nomenclature à partir des tranches alphabétiques délimitées par des segments de deux à quatre lettres de l'interface de consultation est mise en échec à la fois par des lacunes diverses affectant certaines lettres $(A, B, O \ldots)$ et par l'interclassement d'items français et anglais (exemple de séquence, dans la tranche Ba-Bak: bactériologiste, bacteriology, bactériophage). Des sondages témoignent d'actualisations limitées de la nomenclature (covoiturage, smartphone, sudoku, mais pas écoparticipation, écotaxe, hameçonnage, hashtag, phablette, relou, selfie, vapoter), dont la portée ne serait évaluable que par une étude méthodique.

- Le Dictionnaire français de L'Internaute

L'origine du DF-LInt, auquel on accède par la fenêtre de saisie en haut à droite des pages ou en choisissant « Dictionnaire » dans le menu « Plus » sur linternaute.com, site d'actualités et de loisirs de CCM Benchmark Group (Groupe Figaro), n'est pas non plus documentée, mais on peut évaluer sa nomenclature à environ 83000 items grâce à un accès à celle-ci par segments alphabétiques de deux lettres, à partir de chaque lettre ou du lien vers l' "Abécédaire ». Cette valeur élevée est corroborée par les sondages d'évaluation de son actualisation continue, qui attestent l'intégration de chelou, covoiturage, écoparticipation, écotaxe, flashcode, hameçonnage, hashtag, phablette, QR code, relou, selfie, smartphone, sudoku, vapoter ou wesh (interjection), mais pas, cependant, celle de box (féminin) ou vénère. L'interface de consultation propose malcommodément les résultats des requêtes par saisie dans des listes interclassant des occurrences des motifs dans des pages du site et des annonces sur Google. La présentation des données combine, elle, une succession linéaire avec étiquetage de certaines rubriques («Féminin » et « Pluriel» pour les flexions, «Sens » (numérotés), « Exemple », «Synonyme(s) » et également « Traduction anglais » pour les mots, et « Sens » et « Origine» pour les expressions) et l'accès par des boutons à deux de celles-ci (« Définitions », « Synonyme(s) ») et à deux autres (« Conjugaison», «Expressions »). Trois regroupements thématiques de sous-ensembles d'adresses (cf. § 4) sont en outre accessibles dans des bandeaux en haut et en bas de la page d'accueil et en bas des pages consultées : "Les thèmes » (dénombrant et listant les items ressortissant à quatre-vingt-sept domaines d'activité), « Les usages » (pour ceux relevant de huit registres ou d'emplois figurés) et «Les types de mots » (pour la distribution du lexique en cinquante et une catégories grammaticales).

- Le dictionnaire La-definition.fr

La-definition.fr [Ld.f] sur la-definition.fr, sous-titré L'orthographe pour tous et dépourvu de mentions légales, donne accès, par des fenêtres de saisie et des liens, à des «Définitions » (pour «plus de 35000 mots répertoriés »), des « Synonymes » (pour « 7000 mots ») et des 
« Conjugaisons » («plus de 7000 verbes conjugués »), mais rien n'indique si ces valeurs modestes réfèrent aux articles spécifiques du site, de facture sommaire, ou incluent ceux pris dans plusieurs dictionnaires libres de droits plus ou moins anciens (différentes éditions du $D A F$, dont une partie du DAF9, le Féraud, le Littré...). En tout état de cause, l'actualité de sa couverture lexicale est médiocre : s'il connaît ordinateur et virus (informatique), il ignore tous les mots récents utilisés pour sonder les nomenclatures des dictionnaires en ligne, mais aussi des mots plus anciens (abribus, cha-cha-cha, décaféiné, gérontologie, rock'n'roll...).

\subsection{Dictionnaires collaboratifs / participatifs et apparentés}

Six dictionnaires, sur six sites, émanent de la mouvance collaborative / participative.

- Le Wiktionnaire

Sous-titré Le dictionnaire libre, le Wiktionnaire [Wk] consultable sur fr.wiktionary.org est, depuis 2004, la composante francophone du projet multilingue Wiktionary, développé par une communauté de contributeurs au sein de la Wikimedia Foundation, à but non lucratif. Il est bien documenté, sur son propre site et dans l'article que lui consacre Wikipédia, tant quant à l'ordre de grandeur élevé de sa nomenclature, via ses statistiques régulièrement actualisées («328969» lemmes français le 20 décembre 2018, noms propres et locutions compris, et «371019» le 20 mars 2019), qu'à l'organisation de ses articles, de facture classique et à vocation documentaire étendue (une vingtaine de rubriques possibles inventoriées dans l'article de Wikipédia). D'abord nourri de ressources dictionnairiques libres plus ou moins anciennes (notamment le $D A F 8$ ), il est désormais bien actualisé, en dépit du caractère par essence aléatoire de son enrichissement, et fait partie des dictionnaires qui prennent le plus en compte - attestations à l'appui - la diversité des innovations lexicales, notamment celles qui relèvent des variations diastratique et diaphasique : à l'inventaire de celles du DF-LInt (cf. $\S 5.3)$, le Wk ajoute ainsi à l'arrache, code QR, seum, vénère, yes (interjection) ou yo.

- Le Webtionnaire

Le Webtionnaire $[W b]$ sur webtionnaire.fr, développé en Finlande par Sanakirja.org Solutions Oy («Contacts ») et aussi dénommé Dictionnaire-Libre (ibid.), est explicitement «basé sur les articles de Wiktionnaire » (vers lequel redirige un lien). Dans une interface traductionnelle épurée où le choix du français comme langues source et cible limite les données affichées à la composante monolingue, il adapte, dans une disposition semi-tabulaire, les éléments les plus fonctionnels des articles sources : définitions (le cas échéant déhiérarchisées), sélection ou ajout d'exemples (dissociés de celles-ci et sans références), flexion, appartenance à des catégories (registrales, lexicales, étymologiques, flexionnelles, grammaticales), synonymes et prononciation (dans cet ordre, de haut en bas et de gauche à droite). L'actualisation est proche de celle du Wk, seuls code $Q R$ / $Q R$ code, wesh (interjection) et yo faisant défaut parmi les mots testés.

- OmegaWiki

De même inspiration que le Wiktionary (internationalité, utilisation de données libres, collaborativité), le projet OmegaWiki, sur omegawiki.org, répond à une ambition et utilise une méthodologie différentes : «créer un dictionnaire de tous les mots de toutes les langues, incluant des informations lexicales, terminologiques et ontologiques », en stockant les données dans une base relationnelle structurée à partir de concepts pour faciliter les traductions et permettre un affichage plurilingue des résultats des requêtes. L'avancement est moins poussé, les données actuelles reposant d'abord sur le GEMET (GEneral Multilingual Environmental Thesaurus), ressource produite par l'Agence européenne pour l'environnement qui compile différents vocabulaires multilingues, à laquelle « quelques autres définitions [...] ont commencé à être rajoutées par des contributeurs ». Ses statistiques sont de fait modestes («30819» concepts définis début 2019 pour le lexique français, « $31561 »$ le 7 avril 2020) et son actua- 
lisation est en devenir : des mots récents déjà évoqués, seuls hameçonnage, hashtag, smartphone, sudoku et yes sont intégrés à sa nomenclature et définis en français.

- Le dictionnaire français d'educalingo

Les informations manquent concernant le $d f$-educa (sous-titré Le dictionnaire pour les personnes curieuses), qu'on peut consulter sur educalingo.com. Le français n'y est qu'une des vingt langues prises en charge, et les « Conditions générales d'utilisation » accessibles en bas d'écran (icône « (C) »), seul document évoquant la source des articles, ne fournissent, dans des formulations inégalement idiomatiques, que d'assez sommaires renseignements : spécification que « [1] a plupart des articles assortant [sic] chaque définition sont tirés de Wikipedia» (ce qui peut surprendre pour un dictionnaire) « et sont distribués sous licence creative commons », incitation à " citer educalingo.com comme source [en cas de réemploi] afin de manifester [sa] reconnaissance aux personnes qui, grâce à leurs efforts, rendent possible ce projet », et remerciements à «toutes les personnes et organisations qui de manière désintéressée collaborent avec [sic] ce projet ». Évaluée à trois millions de mots pour l'ensemble de ses langues et décrite comme «toujours en augmentation » par la présentation (« À propos de educalingo »), elle aussi accessible en bas d'écran (icône «?»), la nomenclature comporte, pour le français, un peu plus de 87000 items, inventoriables via l'index, vers lequel mène un lien sous la fenêtre de saisie et qui permet l'accès aux articles à partir de séquences initiales de trois lettres. Cette nomenclature, qui intègre des formes nominales fléchies au féminin, paraît peu actualisée, puisque n'y figurent, parmi les mots récents déjà évoqués, que $Q R$ Code et smartphone (traduits du dictionnaire anglais), et que certains manques de Ld.f (cf. § 5.3) s'y observent aussi (abribus). Conformément à la volonté affirmée dans la présentation de proposer « une conception amusante, plus intuitive et remplie de graphiques et d'images » des dictionnaires, la microstructure est une suite de rubriques hétéroclites titrées, dont certaines sont originales (cf. § 4) mais présentent des données invérifiables : «prononciation », « catégorie grammaticale », « définition », « conjugaison », « mots rimant avec » ou « commençant comme » ou « finissant comme » le mot adresse, « synonymes », « antonymes », nuage de «mots en rapport avec » le mot adresse, «traduction » dans vingt-cinq langues, «tendances d'usage du terme » à travers le monde, «fréquence d'utilisation » depuis l'an 1500, occurrences dans diverses sources textuelles, le tout entrecoupé de publicités.

\section{- L'Online Dictionary d'Ultralingua}

La présentation («About us ») de l'Online Dictionary $[O D]$ sur ultralingua.com faisait état d'une équipe pluridisciplinaire («software developpers, designers, linguists and researchers ») créant et maintenant des logiciels, et notamment des dictionnaires. Selon l'article Ultralingua du Wikipedia anglais, ce collectif se serait constitué dans le Minnesota à partir de 1996 autour d'un groupe de professeurs du Carleton College. L'interface de l'OD, modifiée depuis, était celle d'un dispositif bilingue dans lequel, comme sur le site sensAgent - traduction (cf. § 5.2.2), il fallait choisir le français comme langues source et cible (ce qui n'est plus possible en décembre 2019) pour accéder, sous « Online Dictionary », aux articles d'un dictionnaire monolingue dont les sources documentaires n'étaient pas indiquées et qui proposaient, dans le cas optimal, catégorisation, prononciation, définitions, exemples, homonymes et expressions. En dépit de ce qu'affirmait la présentation, l'actualisation de la nomenclature semblait médiocre, à en juger par le nombre restreint et l'ancienneté relative des items déjà évoqués recensés (abribus, cha-cha-cha, décaféiné, écotaxe, gérontologie, ordinateur). La libre consultation s'interrompait au bout d'une dizaine de requêtes, la création d'un compte étant alors requise, mais elle pouvait être relancée plus tard, dans les mêmes limites.

- Le dictionnaire multilingue de Glosbe

Une interface traductionnelle du même ordre est proposée par Glosbe - le dictionnaire multilingue en ligne $[G-D M L]$ sur glosbe.com, site collaboratif qui exploite une "mémoire de traduction » à base principalement de «corpus parallèles » et qui accueille « des milliers de dictionnaires » pour couvrir « presque toutes les langues existantes ». La documentation four- 
nie par ses pages d'accueil générale et du dictionnaire « français - français », dont l'idiomaticité est approximative, et par les pages «A propos de ce site» et « Partners and contributors » accessibles depuis les liens «A propos de nous » et «Partenaires » de la page d'accueil française, évoque une grande communauté de collaborateurs pilotée par deux programmeurs polonais et mentionne diverses ressources utilisées, parmi lesquelles on trouve notamment Wiktionary project et OmegaWiki. L'ordre de grandeur des ressources est supérieur au milliard de phrases pour l'ensemble des dictionnaires. Pour le français, la rubrique «Statistiques » concaténait deux valeurs difficiles à interpréter : «Il y a actuellement 2084170 phrases traduites. » et « Actuellement nous avons 5729350 phrases traduites ». Linéaires, les articles proposent, dans le meilleur des cas (par exemple s.v. vieux), une prononciation vocale et transcrite, des définitions, des synonymes, la possibilité d'accéder aux flexions et des occurrences en mémoire de traduction avec mise en relief de l'item illustré et possibilité d'identifier les sources grâce à des icônes cliquables, à quoi s'ajoutent des suggestions de lemmes pour les requêtes portant sur des formes fléchies. La plupart des mots testés pour appréhender l'actualisation des dictionnaires en ligne sont répertoriés (manquaient écoparticipation et phablette, mot maintenant intégré), mais avec des traitements différenciés par des combinaisons diverses de composants : prononciation vocale, définition, flexion et occurrences pour covoiturage et smartphone ; définition, flexion et occurrences pour hameçonnage et sudoku ; flexion et occurrences pour chelou, hashtag, relou, selfie, seum, vapoter, vapoteur (une occurrence), vapoteuse et wesh (interjection) ; flexion pour box (féminin) et vénère ; occurrences pour à l'arrache, code $Q R$ et $Q R$ code, écotaxe, flashcode, vapotage, yes et yo.

\subsection{Dictionnaire didactique natif}

Dernier dictionnaire repéré, présent sur un seul site et singulier par sa vocation didactique : l'Interactive Language Toolbox [ILT] (anciennement Dictionnaire d'apprentissage du français langue étrangère ou seconde) sur ilt.kuleuven.be/inlato, ancré dans un site universitaire destiné à des étudiants néerlandophones apprenant le français et/ou l'anglais, avec pour conséquence qu'il n'apparaît pas dans les réponses aux requêtes lexicales aléatoires sur Internet (cf. § 2). La question de l'actualisation ne se pose pas pour sa nomenclature, délibérément limitée aux 5000 lemmes et aux 6731 vocables considérés comme les plus fréquents (cf. Verlinde, Selva \& Binon 2009 : 219). Si son site de consultation ne propose pas de métadonnées, ses spécificités sont connaissables par diverses publications paratextuelles ayant Jean Binon et Serge Verlinde comme auteurs pivots : documentation dans des corpus journalistiques, description syntaxique et sémantique des emplois inspirée de modèles linguistiques contemporains et conjoignant définitions syntagmatiques et phrastiques, pluralité des modes d'interrogation, pour la compréhension et surtout l'expression, exploitant une base de données relationnelle.

\section{Bilan provisoire}

Provisoire est le maître mot de cette contribution : «Diversité de l'inventaire provisoire » $(\S 4)$, «Classement provisoire » $(\S 5)$, et maintenant « Bilan provisoire ». C'est que rien n'est stable sur Internet : entre mi-octobre 2018 et fin mars 2019, le site tutélaire de l'Académie française s'est ajouté à celui de l'ATILF, avec une interface nouvelle, comme espace de consultation du $D A F 9$ (§ 5.1.1), le site propre du $M d$ semble avoir disparu au profit de celui de Notre famille ( $\$ 5.3)$, le DCor sur cordial.fr/dictionnaire/ a connu une augmentation de $41 \%$ du nombre de ses entrées et de $63 \%$ de celui de ses définitions ( $\$$ 5.2.1) et la nomenclature du $W k$ s'est également accrue significativement ( $\$ 5.4)$; et d'autres changements sont intervenus depuis : suppression de l'interface unilingue de l'OD $(\$ 5.4)$ et surtout investissement récent de l'offre gratuite par Usito (payant jusqu'en octobre 2019) et par le Dico en ligne Robert (avril 2020). 
L'amplitude de certaines évolutions, concernant notamment l'ordre de grandeur déclaré de nomenclatures, est sans commune mesure avec la modestie des changements opérés au fil des années dans l'espace contraint des plus grands monovolumes qui occupent aujourd'hui le sommet de l'offre imprimée (cf. § 1). Même avec les réserves suscitées par la difficulté de connaître les contenus lexicaux impliqués ou par le fait que des variations flexionnelles donnent lieu à des dédoublements d'entrées (par exemple les formes masculine et féminine de noms d'agent dans le DCor), on peut penser que la lexicographie électronique du français a pris, sur certains sites, l'essor quantitatif permis par le support qui est annoncé comme possible depuis plusieurs décennies.

En matière d'enrichissement des nomenclatures par leur actualisation, quelques dictionnaires, que diverses choses séparent, se détachent, à des rythmes différents : le $W k$ et le $G-D M L$, collaboratifs, le DCor, taliste, le DF-LInt, d'origine indéterminée, et le DdF-Lar, éditorial. Mais d'autres - TLFi, GLLF - quittent chaque jour un peu plus le présent pour l'histoire (cf. $§ 3$ ).

Ce défaut de maintenance est d'autant plus regrettable que ce sont ces dictionnaires qui présentent les contenus descriptifs les plus riches, avec, sur des échelles et selon des modalités différentes, le $D A F 9$ et l'ILT, alors que les traitements microstructurels des répertoires gratuits en ligne sans corrélat imprimé sont couramment minimalistes et parfois indigents, le $W k$ faisant ici exception avec le nombre élevé de ses rubriques activables et la dimension philologique de ses attestations exploitant des sources imprimées et électroniques (cf. § 5.4).

La conception même des dictionnaires est globalement on ne peut plus conventionnelle, l'ILT seul sortant cette fois du lot en conjuguant les apports descriptifs de plusieurs courants linguistiques, certains dispositifs de dictionnaires britanniques pour apprenants allophones et l'exploitation méthodique des possibilités d'interrogation de la base de données sous-jacente via une interface interactive $(\$ 5.5)$.

Les interfaces de consultation, justement, sont souvent datables par leur esthétique, et par là même datées, et sur plusieurs d'entre elles le repérage des données lexicographiques est diversement troublé par les annonces commerciales qui financent les sites. Dans ceux qui sont exempts de publicité, la nouvelle présentation du DAF9 sur dictionnaire-academie.fr se signale par son graphisme rénové et son adaptabilité à tous les écrans ( $\$ \S 4$ et 5.1.1), qui propulsent à la pointe de l'affichage électronique un dictionnaire ancré dans une tradition plus que triséculaire et voué à enregistrer les usages lexicaux les plus confirmés.

Même s'il a tenté de couvrir la globalité de l'offre de dictionnaires généraux du français "actuel" gratuits en ligne à un moment déterminé, le présent repérage n'est qu'un point de départ possible pour de nécessaires approfondissements, tant quant à l'évaluation des nomenclatures, appuyée ici seulement sur un petit nombre de sondages empiriques, que concernant l'analyse qualitative des informations fournies, qui n'a même pas été esquissée.

Merci à Rémi Cardon, Laurent Catach, Noé Gasparini, Franck Sajous et Camille Wadoux pour leurs apports documentaires.

\section{Références bibliographiques}

\section{Dictionnaires, encyclopédies, bases et logiciels}

Base de données lexicographiques panfrancophone. http://www.bdlp.org/.

Cordial 21 Pro / Référence. Logiciels téléchargeables pour PC. Toulouse : Synapse Développement. 2016.

Dico en ligne. https://dictionnaire.lerobert.com/.

Dictionnaire de français. Paris : Larousse. Application pour iOS 8.0 et ultérieurs. Version 3.3. 2016. - Application pour Android. Mise à jour 2018. [DdF-Lar] 
Dictionnaire de l'Académie française. $8^{\mathrm{e}}$ éd. 2 vol. Paris : Librairie Hachette. 1932-1935. [DAF8] - 9e éd. 3 vol. parus (A-Enz, Éoc-Map, Maq-Quo). Paris : Imprimerie Nationale Éditions (et Librairie Arthème Fayard pour les tt. 2 et 3). 1992, 2000, 2011. [DAF9]

Dictionnaire Hachette langue française. Paris : Hachette Éducation. 2001.

Féraud, abbé J.-F. (1787-1788). Dictionnaire critique de la langue française. 3 vol. Marseille : Jean Mossy. [Féraud]

FranceTerme. http://www.culture.fr/franceterme.

Grand Larousse de la langue française en sept volumes. Paris : Librairie Larousse. 1971-1978. - Réimpression. Larousse. 1989. [GLLF]

Grand Larousse en 5 volumes. Paris : Larousse. 1987.

Grand usuel Larousse. 5 vol. Paris : Larousse. 1997.

Le Grand Robert de la langue française. Nouv. éd. Paris : Le Robert. 2018. Abonnement en ligne et logiciel téléchargeable pour Mac et PC. [GRLF]

Le Petit Larousse illustré 2019. Paris : Larousse. 2018. - Le Petit Larousse 2019. Application pour iOS 9.0 et ultérieurs. Version 1.2.1. Mise à jour 2019. 2018. [PLI]

Le Petit Robert de la langue française. Éd. 2019. Paris : Le Robert. 2018. - Abonnement en ligne et logiciel téléchargeable pour Mac et PC. - Application pour iOS 7.1 et ultérieurs. Mise à jour 2019. 2018. $[P R]$

Le Robert correcteur. Logiciel téléchargeable pour Mac et PC. Nouv. version. Paris : Le Robert. 2016.

Le Robert illustré 2019 \& son dictionnaire en ligne. Paris : Le Robert. 2018. [RI]

Lexis. Dictionnaire de la langue française. Paris : Librairie Larousse. 1975. - Tirage le plus récent: Le Lexis. Le dictionnaire érudit de la langue française. Larousse. 2014.

Littré, É. (1863-1873). Dictionnaire de la langue française. 4 vol. Paris : Librairie Hachette et Cie. [Littré]

Maxipoche 2014. Paris : Larousse. 2013. [Mp]

Trésor de la langue française. Dictionnaire de la langue du XIX et du XX ${ }^{e}$ siècle (1789-1960). 16 vol. Paris : Éditions du Centre National de la Recherche Scientifique (tt. 1-10) / Gallimard (tt. 1116). 1971-1994. [TLF]

Usito. https://usito.usherbrooke.ca/.

Wikipédia. L'encyclopédie libre. https://fr.wikipedia.org/wiki/Wikip\%C3\%A9dia:Accueil_principal.

\section{Autres références}

Corbin, F. \& Corbin, P. (2008). Les dictionnaires sont des voitures comme les autres. In Maniez, F. \& Dury, P. (dir.). Lexicographie et terminologie : histoire de mots. Hommage à Henri Béjoint. Gap : Louis Jean Imprimeur. 47-65.

Corbin, P. (1991). Le maquis lexicographique. Aperçus sur l'activité lexicographique monolingue dans le domaine français à la fin du $\mathrm{XX}^{\mathrm{e}}$ siècle. Le français aujourd'hui, 94, 6-26.

Corbin, P. (1998). La lexicographie française est-elle en panne ? In Cicle de Conferències 96-97. Lèxic, corpus i diccionaris. Barcelona : Institut Universitari de Lingüística Aplicada, Universitat Pompeu Fabra. 83-112.

Corbin, P. (2002). Lexicographie et linguistique : une articulation difficile. L'exemple du domaine français. In Melka, F. \& Celeste Augusto, M. (eds). De la lexicologie à la lexicographie / From Lexicology to Lexicography. Utrecht : Utrecht Institute of Linguistics OTS. 8-37.

Corbin, P. (2008). Quel avenir pour la lexicographie française ? In Durand, J., Habert, B. \& Laks, B. (éds). Congrès mondial de linguistique française. Paris, 9-12 juillet 2008. Paris : Institut de Linguis- 
tique Française / EDP Sciences. 1227-1250. https://www.linguistiquefrancaise.org/articles/cmlf/abs/ 2008/01/cmlf08352/cmlf08352.html.

Dire, ne pas dire. http://www.academie-francaise.fr/dire-ne-pas-dire.

Gasiglia, N. (à paraitre a). Identification des articles dictionnairiques non référencés fournis par google. fr. Études de linguistique appliquée.

Gasiglia, N. (à paraître b). Étude généalogique d'articles dictionnairiques affichés par Google. In actes du colloque Au carrefour des sens - IV édition (Uniwersytet Wrocławsky, 26-27 septembre 2019).

Rey-Debove, J. (1971). Étude linguistique et sémiotique des dictionnaires français contemporains. The Hague / Paris : Mouton.

Verlinde, S., Selva, T. \& Binon, J. (2009). Les bases de données au service d'un dictionnaire d'(auto-) apprentissage pour allophones. Lexique, 19, 217-233.

\section{Annexes : URL des sites métadictionnairiques et dictionnairiques actives début 2019}

\section{Annexe 1 : Sites ciblant des sites de consultation de dictionnaires généraux du français "actuel" (par ordre décroissant du nombre des dictionnaires perti- nents ciblés, listés dans l'ordre de leur présentation au § 5)}

- https://www.lexilogos.com/francais_dictionnaire.htm

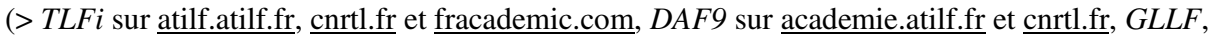

$D d F$-Lar sur larousse.fr et fracademic.com, $M p, G R L F, P R, d-K, D C o r$ sur cordial.fr/dictionnaire/ et dictionnaire.reverso.net, $s A$ sur traduction.sensagent.com et dictionnaire.sensagent.leparisien.fr, $M d, D F$-LInt, Wk, df-educa)

- https://www.lexicool.com/dictionnaire-ressources-francais.asp

( $>$ TLFi sur cnrtl.fr, $D A F 9$ sur cnrtl.fr et dictionnaire-academie.fr, $D d F$-Lar sur larousse.fr, $D C o r$ sur dictionnaire.reverso.net (bouton « Collins »))

- https://www.lepointdufle.net/p/dictionnaire.htm\#premier

( $>T L F i$ sur cnrtl.fr, $D A F 9$ sur cnrtl.fr et dictionnaire-academie.fr, $D d F$-Lar sur larousse.fr, $D C o r$ sur dictionnaire.reverso.net, $s A$ sur dictionnaire.sensagent.leparisien.fr)

- http://www.les-dicos.com/dictionnaire-francais.html

( $>T L F i$ sur atilf.atilf.fr, $D A F 9$ sur academie.atilf.fr, $L D, d . n$ )

- http://www.les-dictionnaires.com/francais.html

(>TLFi sur atilf.atilf.fr, DAF9 sur academie.atilf.fr, $L D, d . n$ )

- https://www.alphadictionary.com/directory/Languages/Romance/French/

( $>T L F i$ sur atilf.atilf.fr, $L D, D F-L I n t)$

- http://dictionnaire-mots.com/francais.html

( $>T L F i$ sur atilf.atilf.fr, $L D, W k$ )

- https://dictionnaire.com/

( $>D d F$-Lar sur larousse.fr, $D C$ or sur universalis.fr)

- http://www.yourdictionary.com/languages/romance.htm

( $>D A F 9$ sur academie-francaise.fr, $L D$ )

- https://dvlf.uchicago.edu/

(>TLFi sur cnrtl.fr)

- http://dicoweb.levillage.org/

( $>D A F 9$ sur academie-francaise.fr)

- http://www.le-dictionnaire.com/annuaire/francais.html $(>d . n)$

- http://www.cocoledico.com/

( $>$ import d'articles d'un état non actualisé de $W k$ ) 
- http://www.les-definitions.com/ et http://www.la-definition.com/ ( $>$ recherche de motifs sur Internet menant à des URL de sites dictionnairiques via le moteur de recherche du navigateur)

\section{Annexe 2: Pages de Wikipédia inventoriant et/ou ciblant des sites de consul- tation de dictionnaires généraux du français "actuel"}

- https://fr.wikipedia.org/wiki/Dictionnaire_en_ligne [exemples de dictionnaires en ligne]

- https://fr.wikipedia.org/wiki/Wikip\%C3\%A9dia:Dictionnaires_disponibles_sur_la_Toile (surtout le $\S$ 1.1.1. : « Dictionnaires français contemporains ») [listes de dictionnaires en ligne]

- https://fr.wikipedia.org/wiki/Cat\%C3\%A9gorie:Dictionnaire_en_ligne [index de dictionnaires en ligne]

\section{Annexe 3 : Sites de consultation de dictionnaires généraux du français "ac- tuel" (par ordre de traitement au § 5)}

1. Trésor de la langue française informatisé [TLFi] (§ 5.1.1)

- sur atilf.atilf.fr : $T L F i$

http://atilf.atilf.fr/ [accueil]

http://www.atilf.fr/spip.php?rubrique77 [informations sur le $T L F$ et le $T L F i$, avec des liens vers des listes d'abréviations et de références]

http://atilf.atilf.fr/dendien/scripts/tlfiv4/showps.exe?p=combi.htm;java=no; [interface de consultation]

http://www.atilf.fr/spip.php?article917 [informations sur le supplément]

http://atilf.atilf.fr/scripts/mep.exe?CRITERE=SUPPLEMENT;ONGLET=tlfi;OO1=1;OO2=2;ISI

$\mathrm{S}=$ isis_tlfi.txt;MENU=menu_tlfi;OUVRIR_MENU=0;ONGLET $=$ tlfi;OO1 $=1 ; \mathrm{OO} 3=-1$

[informations sur le supplément]

- sur cnrtl.fr : $T L F i$

http://www.cnrtl.fr/dictionnaires/modernes/ [accueil et liens vers la présentation et l'accueil du $T L F i$ sur atilf.fr]

http://www.cnrtl.fr/definition/, boutons « Portail lexical » puis « Lexicographie » [interface de consultation de plusieurs ressources, avec index alphabétique et « Liste des formes »]

- sur le-tresor-de-la-langue.fr : Le Trésor de la Langue Française informatisé https://www.le-tresor-de-la-langue.fr/ [accueil, interface de consultation et lien vers l'interface de consultation du TLFi sur atilf.fr]

https://www.le-tresor-de-la-langue.fr/a-propos [informations sur les spécificités de cette adaptation restylée du $T L F i]$

- sur fracademic.com : compilé avec d'autres dictionnaires non titrés sous « Encyclopédie Universelle » http://encyclopedie_universelle.fracademic.com/ [interface de consultation de plusieurs ressources avec index de tranches alphabétiques délimitées par des segments de deux à quatre lettres]

2. Dictionnaire de l'Académie française, $9^{\mathrm{e}}$ édition [DAF9] (\$ 5.1.1)

- sur academie.atilf.fr : Dictionnaire de l'Académie française https://academie.atilf.fr/9/ [interface de consultation et lien vers l'accueil du site dictionnaireacademie.fr]

http://www.atilf.fr/spip.php?article4341 [lien vers l'accueil]

http://www.atilf.fr/ [lien vers l'accueil]

http://www.atilf.fr/spip.php?rubrique82 [présentation et lien vers l'interface de consultation]

- sur cnrtl.fr : Dictionnaire de l'Académie française

http://www.cnrtl.fr/dictionnaires/modernes/ [liens vers la présentation sur atilf.fr et vers l'interface de consultation sur academie.atilf.fr]

- sur academie-francaise.fr : Dictionnaire de l'Académie française http://www.academie-francaise.fr/le-dictionnaire/la-9e-edition [présentation, interface de consultation et lien vers l'accueil]

http://www.academie-francaise.fr [interface de consultation et lien vers l'accueil] https://www.dictionnaire-academie.fr/ [accueil, liens vers les paratextes et interface de consultation] https://www.dictionnaire-academie.fr/presentation [présentation détaillée] 
https://www.dictionnaire-academie.fr/aide-en-ligne.html [aide]

https://www.dictionnaire-academie.fr/lancement.html [communiqué de presse du 7 février 2019]

http://www.academie-francaise.fr/sites/academie-francaise.fr/files/dossier_de_presse.pdf [dossier de presse de février 2019]

https://www.dictionnaire-academie.fr/prefaces [liens vers les paratextes]

http://www.academie-francaise.fr/le-dictionnaire-les-neuf-prefaces/preface-la-neuvieme-edition [préfaces des 2 premiers volumes imprimés]

http://academie-francaise.fr/le-dictionnaire-la-9e-edition/addenda [addenda aux trois premiers volumes imprimés]

3. Grand Larousse de la langue française $[G L L F](\S 5.1 .2)$

- sur gallica.bnf.fr : Grand dictionnaire des lettres ; 1-7. Grand Larousse de la langue française https://gallica.bnf.fr/services/engine/search/sru?operation=searchRetrieve\&version=1.2\&collapsin $\mathrm{g}=$ disabled\&rk=171674;4\&query=dc.relation\%20all\%20\%22cb373349405\%22 [accès aux sept volumes numérisés]

4. Dictionnaire de français [DdF-Lar] (\$ 5.1.2)

- sur larousse.fr : Dictionnaire de français https://www.larousse.fr/dictionnaires/francais [accueil et interface de consultation] https://www.larousse.fr/index/dictionnaires/francais [index de tranches alphabétiques délimitées par des mots] https://www.larousse.fr/infos/credits [mentions légales et ours du site]

- sur fracademic.com : compilé avec d'autres dictionnaires non titrés sous « Encyclopédie Universelle » (cf. 1.)

http://encyclopedie_universelle.fracademic.com/ [interface de consultation de plusieurs ressources avec index de tranches alphabétiques délimitées par des segments de deux à quatre lettres]

5. Maxipoche $2014[M p](\$ 5.1 .2)$

- sur fr.thefreedictionary.com : Maxipoche Larousse (@ 2013)

https://fr.thefreedictionary.com/ [accueil et interface de consultation de plusieurs ressources]

6. Grand Robert de la langue française $[$ GRLF] (§ 5.1.2)

- sur fracademic.com : compilé avec d'autres dictionnaires non titrés sous « Encyclopédie Universelle » (cf. 1. et 4.)

http://encyclopedie_universelle.fracademic.com/ [interface de consultation de plusieurs ressources avec index de tranches alphabétiques délimitées par des segments de deux à quatre lettres]

7. Petit Robert $[P R](\S 5.1 .2)$

- sur fracademic.com : compilé avec d'autres dictionnaires non titrés sous « Encyclopédie Universelle » (cf. 1., 4. et 6.)

http://encyclopedie_universelle.fracademic.com/ [interface de consultation de plusieurs ressources avec index de tranches alphabétiques délimitées par des segments de deux à quatre lettres]

8. Robert illustré $[R I](\S 5.1 .2)$

- sur google.com : réponse par défaut à des requêtes lexicales https://www.google.com/ [adaptation non titrée d'une version de la composante "de langue" du $R I$ ]

9. Dictionnaire Hachette. Langue française et synonymes (\$ 5.1.2)

- sur fracademic.com : compilé avec d'autres dictionnaires non titrés sous « Encyclopédie Universelle » (cf. 1., 4., 6. et 7.)

http://encyclopedie_universelle.fracademic.com/ [interface de consultation de plusieurs ressources avec index de tranches alphabétiques délimitées par des segments de deux à quatre lettres]

10. dictionnaire sans titre $[d-K](\S 5.1 .2)$

- sur fr.thefreedictionary.com : dictionnaire sans titre (@ 2013) dans une rubrique « Traductions » https://fr.thefreedictionary.com/ [accueil et interface de consultation de plusieurs ressources, cf. 5.] https://www.lexicala.com/ [accueil du site de la société K Dictionaries]

11. Dictionnaire Cordial [DCor] (\$ 5.2.1)

- sur cordial.fr/dictionnaire/ : Dictionnaire Cordial http://www.cordial.fr/dictionnaire/ [accueil et interface de consultation] 
http://synapse-developpement.fr/ [présentation de la société Synapse Développement]

- sur la-conjugaison.nouvelobs.com : Définition avec Cordial Dico http://la-conjugaison.nouvelobs.com/definition/ [accueil et interface de consultation]

- sur universalis.fr : Dictionnaire avec Cordial Dico

https://www.universalis.fr/dictionnaire/ [accueil, interface de consultation et accès, par le choix de

«Dictionnaire » dans le menu, à une fenêtre surgissante qui les duplique]

https://www.universalis.fr/alpha-dictionnaire/A/ [début de l'index alphabétique de la nomenclature]

- sur dictionnaire.reverso.net : Cordial Dico Dictionnaire Français Définition

https://dictionnaire.reverso.net/francais-definition/ [accueil et interface de consultation]

https://dictionnaire.reverso.net/CollabDict.aspx ?srcLang=1036\&targLang=-2\&word=\&post=1

[interface de consultation du dictionnaire collaboratif]

12. sensAgent $[$ sA $](\S 5.2 .2)$

- sur traduction.sensagent.com : sensAgent - traduction

http://traduction.sensagent.com [accueil plurilingue général et interface de consultation du site] http://traduction.sensagent.com/\%20/fr-fr/ [interface de consultation des ressources françaises] http://www.sensagent.com/common/online-2008/popup/PopUp-informationcompany.fr.html [présentation de la société Sensegates]

- sur dictionnaire.sensagent.leparisien.fr : sensAgent - dictionnaire http://dictionnaire.sensagent.leparisien.fr/index.html [accueil plurilingue général et interface de consultation des ressources françaises]

$\mathrm{http} / / /$ dictionnaire.sensagent.leparisien.fr/dictionnaire/fr-fr/ [interface de consultation des ressources françaises]

http://www.sensagent.com/common/online-2008/popup/PopUp-informationcompany.fr.html [présentation de la société Sensegates, cf. site traduction.sensagent.com]

- sur tv5monde.com : Traducteur multilingue Définitions et traductions avec Alexandria $\mathrm{http} / / / \mathrm{www} . t v 5 \mathrm{monde} . \mathrm{com} / \mathrm{cms} /$ chaine-francophone/outils/p-7550-Traducteur-Alexandria.htm? $\mathrm{sl}=\mathrm{fr} \&$ terme $=\& \mathrm{tl}=\mathrm{fr} \& \mathrm{ok} . \mathrm{x}=0 \& \mathrm{ok} . \mathrm{y}=0$ [accueil plurilingue général et interface de consultation des ressources françaises] http://www.memodata.com/ [présentation des prestations de la société Memodata]

- sur tv5monde.com : Rechercher un mot dans le dictionnaire http://www.tv5monde.com/ [accueil du site et accès au dictionnaire par une icone] https://langue-francaise.tv5monde.com/decouvrir/dictionnaire [interface de consultation]

13. Le Dictionnaire $[L D](\S 5.2 .3)$

- sur le-dictionnaire.com : Le Dictionnaire http://www.le-dictionnaire.com/ [interface de consultation] http://www.le-dictionnaire.com/dico.html [informations et interface de consultation] https://www.societe.com/societe/sarl-blue-painter-402063754.html [présentation de la société Blue Painter]

14. dictionnaire.net $[$ d.n $](\S 5.2 .3)$

- sur dictionnaire.net : dictionnaire.net http://www.dictionnaire.net/ [interface de consultation] http://www.dictionnaire.net/cgu.html [informations] https://www.societe.com/societe/sarl-blue-painter-402063754.html [présentation de la société Blue Painter, cf. 13.]

15. Dictionnaire Mediadico $[$ Md $]$ ( $\$ 5.3)$

- sur notrefamille.com : Définition et Synonymes - Définition Mediadico https://www.notrefamille.com/dictionnaire/ [accueil et interface de consultation de plusieurs ressources] https://fr.wikipedia.org/wiki/Mediadico [article Mediadico de Wikipédia]

16. Dictionnaire français [DF-LInt $](\S 5.3)$

- sur linternaute.com : Dictionnaire français https://www.linternaute.fr/dictionnaire/fr/ [accueil et interface de consultation] 
17. La-définition.fr $[\boldsymbol{L d}$.f $](\S 5.3)$

- sur la-definition.fr : La-definition.fr http://www.la-definition.fr/ [accueil et interface de consultation]

18. Wiktionnaire $[W k](\S 5.4)$

- sur fr.wiktionary.org : Wiktionnaire Le dictionnaire libre https://fr.wiktionary.org/wiki/Wiktionnaire:Page_d\%E2\%80\%99accueil [accueil et interface de consultation]

https://fr.wiktionary.org/wiki/Wiktionnaire:\%C3\%80_propos [présentation] https://fr.wiktionary.org/wiki/Wiktionnaire:Statistiques [statistiques actualisées sur les différentes langues traitées en français]

https://fr.wikipedia.org/wiki/Wiktionnaire [article Wiktionnaire de Wikipédia]

https://www.wiktionary.org/ [accueil du projet Wiktionary]

https://fr.wikipedia.org/wiki/Wikimedia_Foundation [article Wikimedia Foundation de Wikipédia] https://meta.wikimedia.org/wiki/Wikim\%C3\%A9dia_France/Groupes_de_travail/Groupes_th\%C 3\%A9matiques/Promotion_et_communication/Communiqu\%C3\%A9s_de_presse/15_ans_du_ Wiktionnaire [communiqué de presse du $15^{\mathrm{e}}$ anniversaire (22 mars 2019) : statistiques]

19. Webtionnaire $[\mathrm{Wb}](\S 5.4)$

- sur http://webtionnaire.fr : Webtionnaire http://webtionnaire.fr/ [accueil et interface de consultation] http://webtionnaire.fr/contact.php [informations et interface de consultation]

20. OmegaWiki (§ 5.4)

- sur omegawiki.org : OmegaWiki

http://www.omegawiki.org/Meta:Main_Page/fr [accueil et interface de consultation]

http://www.omegawiki.org/Portal:fra [présentation et interface de consultation]

http://www.omegawiki.org/Help:Introduction_to_OmegaWiki [présentation générale en anglais et interface de consultation]

https://fr.wikipedia.org/wiki/General_Multilingual_Environmental_Thesaurus [informations sur la source documentaire]

http://www.omegawiki.org/Special:Ow_statistics?showstat=dm [statistiques et interface de consultation]

21. dictionnaire français $[$ df-educa] $(\S 5.4)$

- sur educalingo.com : dictionnaire français https://educalingo.com/fr/dic-fr [accueil, interface de consultation et lien vers l'index] https://educalingo.com/fr/about [présentation] https://educalingo.com/fr/dic-fr/abc [index alphabétique de la nomenclature] https://educalingo.com/fr/legal-advice [conditions d'utilisation]

22. Online Dictionary $[O D](\S 5.4)$

- sur ultralingua.com : Online Dictionary https://www.ultralingua.com/ [accueil et ancienne interface de consultation] https://www.ultralingua.com/about-ultralingua.html [présentation de la société Ultralingua en anglais désormais inactive] https://en.wikipedia.org/wiki/Ultralingua [article Ultralingua de Wikipedia en anglais]

23. Glosbe $[\boldsymbol{G}$-DML] (§ 5.4)

- sur glosbe.com : Glosbe - le dictionnaire multilingue en ligne https://glosbe.com/ [accueil et interface de consultation] https://glosbe.com/about [présentation en anglais] https://glosbe.com/partners [sources]

24. Interactive Language Toolbox $[$ ILT] $(\S 5.5)$

- sur ilt.kuleuven.be : Interactive Language Toolbox https://ilt.kuleuven.be/inlato/ [interface de consultation générale, en néerlandais par défaut] https://ilt.kuleuven.be/inlato/index.php?int=3 [interface de consultation en français] https://www.youtube.com/watch?v=-AHejt2vpx $8 \& h d=1$ [tutoriel en vidéo] https://www.youtube.com/watch?v=Z9yr_fw73fg\&hd=1 [tutoriel en vidéo] 